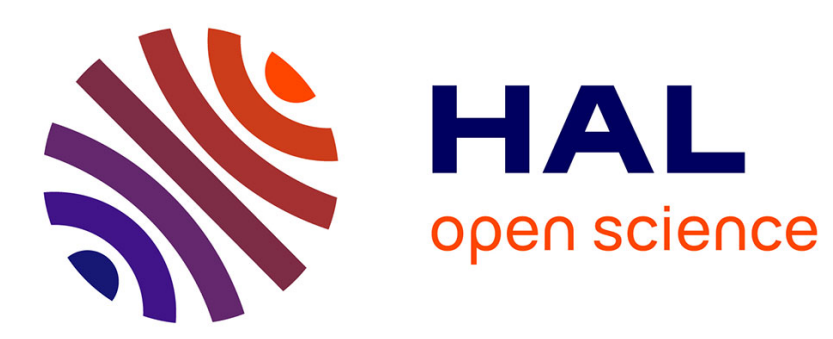

\title{
Perturbation Method for Particle-like Solutions of the Einstein-Dirac-Maxwell Equations
}

\author{
Simona Rota Nodari
}

\section{To cite this version:}

Simona Rota Nodari. Perturbation Method for Particle-like Solutions of the EinsteinDirac-Maxwell Equations. Comptes Rendus. Mathématique, 2010, 348, pp.Pages 791-794. 10.1016/j.crma.2010.06.003 . hal-00442453

\section{HAL Id: hal-00442453 https://hal.science/hal-00442453}

Submitted on 21 Dec 2009

HAL is a multi-disciplinary open access archive for the deposit and dissemination of scientific research documents, whether they are published or not. The documents may come from teaching and research institutions in France or abroad, or from public or private research centers.
L'archive ouverte pluridisciplinaire HAL, est destinée au dépôt et à la diffusion de documents scientifiques de niveau recherche, publiés ou non, émanant des établissements d'enseignement et de recherche français ou étrangers, des laboratoires publics ou privés. 


\title{
PERTURBATION METHOD FOR PARTICLE-LIKE SOLUTIONS OF THE EINSTEIN-DIRAC-MAXWELL EQUATIONS
}

\author{
SIMONA ROTA NODARI
}

\begin{abstract}
The aim of this Note is to prove by a perturbation method the existence of solutions of the coupled Einstein-Dirac-Maxwell equations for a static, spherically symmetric system of two fermions in a singlet spinor state and with the electromagnetic coupling constant $\left(\frac{e}{m}\right)^{2}<1$. We show that the nondegenerate solution of Choquard's equation generates a branch of solutions of the Einstein-Dirac-Maxwell equations.

Une méthode de perturbation pour les solutions localisées des équations d'Einstein-Dirac-Maxwell.

RÉsumé. Le but de cette Note est de démontrer par une méthode de perturbation l'existence de solutions des équations d'Einstein-Dirac-Maxwell pour un système statique, à symétrie sphérique de deux fermions dans un état de singulet et avec une constante de couplage électromagnétique $\left(\frac{e}{m}\right)^{2}<1$. On montre que la solution non dégénérée de l'équation de Choquard génère une branche de solutions des équations d'Einstein-Dirac-Maxwell.
\end{abstract}

\section{VERSION FRANÇAISE ABRÉGÉE}

Dans un papier récent [5], par une méthode de perturbation, on a montré de manière rigoureuse l'existence de solutions des équations d'Einstein-Dirac pour un système statique, à symétrie sphérique de deux fermions dans un état de singulet. Dans cette Note, on généralise ce résultat aux équations d'Einstein-Dirac-Maxwell et on montre, dans le cas particulier d'un couplage électromagnétique faible, l'existence des solutions obtenues numériquement par F. Finster, J. Smoller et ST. Yau dans [7].

Plus précisément, en utilisant l'idée introduite par Ounaies pour une classe d'équations de Dirac non linéaires (voir [1]) et adaptée dans [5] aux équations d'Einstein-Dirac, on obtient le théorème suivant.

Théorème 0.1. Soient e, $m, \omega$ tels que $e^{2}-m^{2}<0,0<\omega<m$ et supposons $m-\omega$ assez petit; alors il existe une solution non triviale de (⿴囗大 15 ).

Dans cette Note, on décrit la méthode utilisée pour démontrer ce théorème. Premièrement, par un changement d'échelle, on transforme les équations d'EinsteinDirac-Maxwell (649) en un système perturbé qui s'écrit sous la forme (11). On choisit $\varepsilon=m-\omega$ comme paramètre de perturbation.

Deuxièment, on remarque que, pour $\varepsilon=0$ et $\left(\frac{e}{m}\right)^{2}<1$, ce système est équivalent au système (12) où l'équation pour la variable $\varphi$ est l'équation de Choquard. Il est bien connu que l'équation de Choquard a une solution radiale positive. De plus, dans l'espace des fonctions radiales, cette solution est non dégénérée, dans le sens où le

Date: December 18, 2009. 
noyau de la linéarisation de l'équation contient seulement la fonction identiquement nulle. On appelle $\phi_{0}$ la solution du système (12).

Ensuite, on observe que le système perturbé s'écrit sous la forme $D(\varepsilon, \varphi, \chi, \tau, \zeta)=$ 0 avec $D$ un opérateur non linéaire de classe $\mathcal{C}^{1}$, pour un bon choix d'espaces fonctionnels. On prouve que cet opérateur satisfait les hypothèses du théorème des fonctions implicites. En particulier, on montre que la linéarisation de l'opérateur $D$ par rapport à $(\varphi, \chi, \tau, \zeta)$ en $\left(0, \phi_{0}\right), D_{\varphi, \chi, \tau, \zeta}\left(0, \phi_{0}\right)$, est une injection, grâce à la non-dégénérescence de la solution de l'équation de Choquard, et s'écrit comme somme d'un isomorphisme et d'un opérateur compact; donc $D_{\varphi, \chi, \tau, \zeta}\left(0, \phi_{0}\right)$ est un isomorphisme. En appliquant le théorème des fonctions implicites, on déduit que, pour $\varepsilon$ assez petit et $e^{2}-m^{2}<0$, le système (11) a une solution.

En conclusion, pour $e^{2}-m^{2}<0,0<\omega<m$ et $m-\omega$ assez petit, les équations d'Einstein-Dirac-Maxwell possèdent une solution non triviale.

\section{INTRODUCTION}

In a recent paper [5], using a perturbation method, we proved rigorously the existence of solutions of the coupled Einstein-Dirac equations for a static, spherically symmetric system of two fermions in a singlet spinor state. In this Note, we extend our result to the Einstein-Dirac-Maxwell equations and we prove, in the particular case of a weak electromagnetic coupling, the existence of the solutions obtained numerically by F. Finster, J. Smoller and ST. Yau in [7].

The general Einstein-Dirac-Maxwell equations for a system of $n$ Dirac particles take the form

$$
(G-m) \psi_{a}=0, \quad R_{j}^{i}-\frac{1}{2} R \delta_{j}^{i}=-8 \pi T_{j}^{i}, \quad \nabla_{k} F^{j k}=4 \pi e \sum_{a=1}^{n} \overline{\psi_{a}} G^{j} \psi_{a}
$$

where $G^{j}$ are the Dirac matrices, $G$ denote the Dirac operator, $\psi_{a}$ are the wave functions of fermions of mass $m$ and charge $e, F_{j k}$ is the electromagnetic field tensor and, finally, $T_{j}^{i}$ is the sum of the energy-momentum tensor of the Dirac particle and the Maxwell stress-energy tensor.

In [7], the metric, in polar coordinates $(t, r, \vartheta, \varphi)$, is given by

$$
d s^{2}=\frac{1}{T^{2}} d t^{2}-\frac{1}{A} d r^{2}-r^{2} d \vartheta^{2}-r^{2} \sin ^{2} \vartheta d \varphi^{2}
$$

with $A=A(r), T=T(r)$ positive functions; moreover, using the ansatz from [6], Finster, Smoller and Yau describe the Dirac spinors with two real radial functions $\Phi_{1}(r), \Phi_{2}(r)$ and they assume that the electromagnetic potential has the form $\mathcal{A}=(-V, 0)$, with $V$ the Coulomb potential. 
In this case the Einstein-Dirac-Maxwell equations can be written as

$$
\begin{aligned}
\sqrt{A} \Phi_{1}^{\prime}= & \frac{1}{r} \Phi_{1}-((\omega-e V) T+m) \Phi_{2} \\
\sqrt{A} \Phi_{2}^{\prime}= & ((\omega-e V) T-m) \Phi_{1}-\frac{1}{r} \Phi_{2} \\
r A^{\prime}= & 1-A-16 \pi(\omega-e V) T^{2}\left(\Phi_{1}^{2}+\Phi_{2}^{2}\right)-r^{2} A T^{2}\left(V^{\prime}\right)^{2} \\
2 r A \frac{T^{\prime}}{T}= & A-1-16 \pi(\omega-e V) T^{2}\left(\Phi_{1}^{2}+\Phi_{2}^{2}\right)+32 \pi \frac{1}{r} T \Phi_{1} \Phi_{2} \\
& +16 \pi m T\left(\Phi_{1}^{2}-\Phi_{2}^{2}\right)+r^{2} A T^{2}\left(V^{\prime}\right)^{2} \\
r^{2} A V^{\prime \prime}= & -8 \pi e\left(\Phi_{1}^{2}+\Phi_{2}^{2}\right)-\left(2 r A+r^{2} A \frac{T^{\prime}}{T}+\frac{r^{2}}{2} A^{\prime}\right) V^{\prime}
\end{aligned}
$$

with the normalization condition $\int_{0}^{\infty}|\Phi|^{2} \frac{T}{\sqrt{A}} d r=\frac{1}{4 \pi}$.

In order that the metric be asymptotically Minkowskian and the solutions have finite (ADM) mass, Finster, Smoller and Yau assume

$$
\lim _{r \rightarrow \infty} T(r)=1
$$

and

$$
\lim _{r \rightarrow \infty} \frac{r}{2}(1-A(r))<\infty .
$$

Finally, they also require that the electromagnetic potential vanishes at infinity.

In this Note, using the idea introduced by Ounaies for a class of nonlinear Dirac equations (see [1]) and adapted in [5] to the Einstein-Dirac equations, we obtain the following result.

Theorem 1.1. Given $e, m, \omega$ such that $e^{2}-m^{2}<0,0<\omega<m$ and $m-\omega$ is sufficiently small, there exists a non trivial solution of (因同).

\section{Perturbation method for the Einstein-DiraC-Maxwell equations}

First of all, we observe that, writing $T(r)=1+t(r)$ and integrating the equation (张), the Einstein-Dirac-Maxwell equations become

$$
\begin{aligned}
\sqrt{A} \Phi_{1}^{\prime}= & \frac{1}{r} \Phi_{1}-((\omega-e V)(1+t)+m) \Phi_{2} \\
\sqrt{A} \Phi_{2}^{\prime}= & ((\omega-e V)(1+t)-m) \Phi_{1}-\frac{1}{r} \Phi_{2} \\
2 r A t^{\prime}= & (A-1)(1+t)-16 \pi(\omega-e V)(1+t)^{3}\left(\Phi_{1}^{2}+\Phi_{2}^{2}\right) \\
& +32 \pi \frac{1}{r}(1+t)^{2} \Phi_{1} \Phi_{2}+16 \pi m(1+t)^{2}\left(\Phi_{1}^{2}-\Phi_{2}^{2}\right) \\
& +r^{2} A(1+t)^{3}\left(V^{\prime}\right)^{2} \\
\sqrt{A}(1+t) V^{\prime}= & -\frac{8 \pi e}{r^{2}} \int_{0}^{r}\left(\Phi_{1}^{2}+\Phi_{2}^{2}\right) \frac{(1+t)}{\sqrt{A}} d s .
\end{aligned}
$$

where $A(r)=1+a(r)$ and

$$
\begin{aligned}
a(r)= & -\frac{1}{r} \exp (-F(r)) \int_{0}^{r}\left[16 \pi(\omega-e V)(1+t)^{2}\left(\Phi_{1}^{2}+\Phi_{2}^{2}\right)\right. \\
& \left.+s^{2}(1+t)^{2}\left(V^{\prime}\right)^{2}\right] \exp (F(s)) d s
\end{aligned}
$$


with $F(r)=\int_{0}^{r} s(1+t)^{2}\left(V^{\prime}\right)^{2} d s$.

After that, we introduce the new variable $(\varphi, \chi, \tau, \zeta)$ such that

$$
\Phi_{1}(r)=\varepsilon^{1 / 2} \varphi\left(\varepsilon^{1 / 2} r\right), \quad \Phi_{2}(r)=\varepsilon \chi\left(\varepsilon^{1 / 2} r\right), \quad t(r)=\varepsilon \tau\left(\varepsilon^{1 / 2} r\right), \quad V(r)=\varepsilon \zeta\left(\varepsilon^{1 / 2} r\right)
$$

where $\Phi_{1}, \Phi_{2}, t, V$ satisfy (6-9) and $\varepsilon=m-\omega$. Using the explicit expression of $a(r)$, given in (10), we write

$$
a\left(\Phi_{1}, \Phi_{2}, t, V\right)=\varepsilon \alpha(\varepsilon, \varphi, \chi, \tau, \zeta)
$$

with $\alpha(0, \varphi, \chi, \tau, \zeta)=-\frac{16 \pi m}{r} \int_{0}^{r} \varphi^{2} d s$. It is now clear that if $\Phi_{1}, \Phi_{2}, t, V$ satisfy (6-9), then $\varphi, \chi, \tau, \zeta$ satisfy the system

$$
\left\{\begin{array}{l}
(1+\varepsilon \alpha(\varepsilon, \varphi, \chi, \tau, \zeta))^{1 / 2} \frac{d}{d r} \varphi-\frac{1}{r} \varphi+2 m \chi+K_{1}(\varepsilon, \varphi, \chi, \tau, \zeta)=0 \\
(1+\varepsilon \alpha(\varepsilon, \varphi, \chi, \tau, \zeta))^{1 / 2} \frac{d}{d r} \chi+\frac{1}{r} \chi+\varphi-m \varphi \tau+e \varphi \zeta+K_{2}(\varepsilon, \varphi, \chi, \tau, \zeta)=0 \\
(1+\varepsilon \alpha(\varepsilon, \varphi, \chi, \tau, \zeta)) \frac{d}{d r} \tau-\frac{\alpha(\varepsilon, \varphi, \chi, \tau, \zeta)}{2 r}+K_{3}(\varepsilon, \varphi, \chi, \tau, \zeta)=0 \\
(1+\varepsilon \alpha(\varepsilon, \varphi, \chi, \tau, \zeta))^{1 / 2}(1+\varepsilon \tau) \frac{d}{d r} \zeta+\frac{8 \pi e}{r^{2}} \int_{0}^{r} \varphi^{2} d s+K_{4}(\varepsilon, \varphi, \chi, \tau, \zeta)=0
\end{array}\right.
$$

where $K_{1}(0, \varphi, \chi, \tau, \zeta)=K_{2}(0, \varphi, \chi, \tau, \zeta)=K_{3}(0, \varphi, \chi, \tau, \zeta)=K_{4}(0, \varphi, \chi, \tau, \zeta)=$ 0.

Then, for $\varepsilon=0$, (11) becomes

$$
\left\{\begin{array}{l}
-\frac{d^{2}}{d r^{2}} \varphi+2 m \varphi+16 \pi\left(e^{2}-m^{2}\right) m\left(\int_{0}^{\infty} \frac{\varphi^{2}}{\max (r, s)} d s\right) \varphi=0 \\
\chi(r)=\frac{1}{2 m}\left(\frac{1}{r} \varphi-\frac{d}{d r} \varphi\right) \\
\tau(r)=8 \pi m \int_{0}^{\infty} \frac{\varphi^{2}}{\max (r, s)} d s \\
\zeta(r)=8 \pi e \int_{0}^{\infty} \frac{\varphi^{2}}{\max (r, s)} d s
\end{array}\right.
$$

We remark that if $e^{2}-m^{2}<0$ the first equation of the system (12) is the Choquard equation

$$
-\triangle u+2 m u-4\left(m^{2}-e^{2}\right) m\left(\int_{\mathbb{R}^{3}} \frac{|u(y)|^{2}}{|x-y|} d y\right) u=0 \quad \text { in } H^{1}\left(\mathbb{R}^{3}\right)
$$

with $u(x)=\frac{\varphi(|x|)}{|x|}$. It is well known that Choquard's equation (13) has a unique radial, positive solution $u_{0}$ with $\int\left|u_{0}\right|^{2}=N$ for some $N>0$ given. Furthermore, $u_{0}$ is infinitely differentiable, goes to zero at infinity and is a radial nondegenerate solution; by this we mean that the linearization of (13) around $u_{0}$ has a trivial nullspace in $L_{r}^{2}\left(\mathbb{R}^{3}\right)$ (see [3], [ [4, [2] for more details).

Let $\phi_{0}=\left(\varphi_{0}, \chi_{0}, \tau_{0}, \zeta_{0}\right)$ be the ground state solution of (12).

The main idea is that the solutions of (11) are the zeros of a $\mathcal{C}^{1}$ operator $D$ : $\mathbb{R} \times X_{\varphi} \times X_{\chi} \times X_{\tau} \times X_{\zeta} \rightarrow Y_{\varphi} \times Y_{\chi} \times Y_{\tau} \times Y_{\zeta}$. So, to obtain a solution of (11) from 
$\phi_{0}$, we define the operators

$$
\begin{aligned}
L_{1}(\varepsilon, \varphi, \chi, \tau, \zeta)= & (1+\varepsilon \alpha(\varepsilon, \varphi, \chi, \tau, \zeta))^{1 / 2} \frac{1}{r} \frac{d}{d r} \varphi-\frac{\varphi}{r^{2}}+2 m \frac{\chi}{r} \\
& +\frac{1}{r} K_{1}(\varepsilon, \varphi, \chi, \tau, \zeta) \\
L_{2}(\varepsilon, \varphi, \chi, \tau, \zeta)= & (1+\varepsilon \alpha(\varepsilon, \varphi, \chi, \tau, \zeta))^{1 / 2} \frac{1}{r} \frac{d}{d r} \chi+\frac{\chi}{r^{2}}+\frac{\varphi}{r}-m \frac{\varphi}{r} \tau+e \frac{\varphi}{r} \zeta \\
& +\frac{1}{r} K_{2}(\varepsilon, \varphi, \chi, \tau, \zeta) \\
L_{3}(\varepsilon, \varphi, \chi, \tau, \zeta)= & (1+\varepsilon \alpha(\varepsilon, \varphi, \chi, \tau, \zeta)) \frac{d}{d r} \tau-\frac{\alpha(\varepsilon, \varphi, \chi, \tau, \zeta)}{2 r}+K_{3}(\varepsilon, \varphi, \chi, \tau, \zeta) \\
L_{4}(\varepsilon, \varphi, \chi, \tau, \zeta)= & (1+\varepsilon \alpha(\varepsilon, \varphi, \chi, \tau, \zeta))^{1 / 2}(1+\varepsilon \tau) \frac{d}{d r} \zeta+\frac{8 \pi e}{r^{2}} \int_{0}^{r} \varphi^{2} d s \\
& +K_{4}(\varepsilon, \varphi, \chi, \tau, \zeta)
\end{aligned}
$$

and

$D(\varepsilon, \varphi, \chi, \tau, \zeta)=\left(L_{1}(\varepsilon, \varphi, \chi, \tau, \zeta), L_{2}(\varepsilon, \varphi, \chi, \tau, \zeta), L_{3}(\varepsilon, \varphi, \chi, \tau, \zeta), L_{4}(\varepsilon, \varphi, \chi, \tau, \zeta)\right)$,

with $X_{\varphi}, X_{\chi}, X_{\tau}, Y_{\varphi}, Y_{\chi}, Y_{\tau}$ defined as in [5] and

$$
\begin{aligned}
X_{\zeta} & =\left\{\zeta:(0, \infty) \rightarrow \mathbb{R} \mid \lim _{r \rightarrow \infty} \zeta(r)=0, \frac{d}{d r} \zeta \in L^{1}((0, \infty), d r) \cap L^{2}((0, \infty), r d r)\right\} \\
Y_{\zeta} & =L^{1}((0, \infty), d r) \cap L^{2}((0, \infty), r d r)
\end{aligned}
$$

with their natural norms.

Next, we linearize the operator $D$ on $(\varphi, \chi, \tau, \zeta)$ around $\left(0, \phi_{0}\right)$ :

$$
\begin{aligned}
& D_{\varphi, \chi, \tau, \zeta}\left(0, \phi_{0}\right)(h, k, l, z)=\left(\begin{array}{c}
\frac{1}{r} \frac{d}{d r} h-\frac{h}{r^{2}}+2 m \frac{k}{r} \\
\frac{1}{r} \frac{d}{d r} k+\frac{k}{r^{2}}+\frac{h}{r}-m \frac{\varphi_{0}}{r} l+e \frac{\varphi_{0}}{r} z \\
\frac{d}{d r} l \\
\frac{d}{d r} z
\end{array}\right) \\
& +\left(\begin{array}{c}
0 \\
-m \frac{h}{r} \tau_{0}+e \frac{h}{r} \zeta_{0} \\
\frac{16 \pi m}{r^{2}} \int_{0}^{r} \varphi_{0} h d s \\
\frac{16 \pi e}{r^{2}} \int_{0}^{r} \varphi_{0} h d s
\end{array}\right)
\end{aligned}
$$

We observe that, thanks to the nondegeneracy of the solution of Choquard's equation, $D_{\varphi, \chi, \tau, \zeta}\left(0, \phi_{0}\right)$ is a one-to-one operator. Moreover, it can be written as a sum of an isomorphism and a compact operator. It is thus an isomorphism. Finally, the application of the implicit function theorem yields the following result, which is equivalent to Theorem 1.1.

Theorem 2.1. Suppose $e^{2}-m^{2}<0$ and let $\phi_{0}$ be the ground state solution of 12), then there exists $\delta>0$ and a function $\eta \in \mathcal{C}\left((0, \delta), X_{\varphi} \times X_{\chi} \times X_{\tau} \times X_{\zeta}\right)$ such that $\eta(0)=\phi_{0}$ and $D(\varepsilon, \eta(\varepsilon))=0$ for $0 \leq \varepsilon<\delta$. 


\section{ACKNOWLEDGEMENTS}

The author would like to thank professor Eric Séré and professor Bernhard Ruf for helpful discussions.

\section{REFERENCES}

[1] H. Ounaies, Perturbation method for a class of non linear Dirac equations. Differential Integral Equations 13 (2000), no. 4-6, 707-720.

[2] E. Lenzmann, Uniqueness of ground states for pseudo-relativistic Hartree equations. Preprint (2008).

[3] E. H. Lieb, Existence and Uniqueness of the Minimizing Solution of Choquard's Nonlinear Equation. Studies in Applied Mathematics 57 (1977), 93-105.

[4] P.L. Lions, The Choquard equation and related questions. Nonlinear Analysis. Theory, Methods and Applications 4 (1980), no. 6, 1063-1073.

[5] S. Rota Nodari, Perturbation Method for Particle-like Solutions of the Einstein-Dirac Equations. Ann. Henri Poincaré (2009), DOI 10.1007/s00023-009-0015-x.

[6] F. Finster, J. Smoller, S.T. Yau, Particlelike solutions of the Einstein-Dirac equations. Physical Review. D. Particles and Fields. Third Series 59 (1999).

[7] F. Finster, J. Smoller, S.T. Yau, Particle-like solutions of the Einstein-Dirac-Maxwell equations. Phys. Lett. A. 259 (1999), no. 6, 431-436.

E-mail address: rotanodari@ceremade.dauphine.fr

Ceremade (UmR CNRS 7534), Université Paris-Dauphine, Place du Maréchal de Lattre de Tassigny, 75775 Paris Cedex 16, France

Dipartimento di Matematica, Università di Milano, Via Saldini 50, 20133 Milan, Italy 